OPEN ACCESS

Edited by:

Thippa Reddy Gadekallu,

VIT University, India

Reviewed by:

Kun Liu,

Beijing Friendship Hospital, China

Zhidong Zhu,

Shanghai Outdo Biotech Co, China

Praveen Kumar,

VIT University, India

*Correspondence:

Chengliang Yin

chengliangyin@163.com

Rilige Wu

wrlg1114@163.com

Qiang Liu

m13992079668@163.com

Xiaoping L

li-xp@zjsru.edu.cn

tThese authors have contributed equally to this work

Specialty section:

This article was submitted to

Digital Public Health,

a section of the journal

Frontiers in Public Health

Received: 12 November 2021

Accepted: 06 December 2021

Published: 06 January 2022

Citation:

Li W, Dong S, Wang B, Wang $H, X u C$,

Zhang K, Li W, Hu Z, Li X, LiU Q, Wu R

and Yin $C$ (2022) The Construction

and Development of a Clinical

Prediction Model to Assess Lymph

Node Metastases in Osteosarcoma.

Front. Public Health 9:813625.

doi: 10.3389/fpubh.2021.813625

\title{
The Construction and Development of a Clinical Prediction Model to Assess Lymph Node Metastases in Osteosarcoma
} Wenle $\mathrm{Li}^{1,2+}$, Shengtao Dong ${ }^{3 \dagger}$, Bing Wang ${ }^{2}$, Haosheng Wang ${ }^{4}$, Chan $\mathrm{Xu}^{2}$, Kai Zhang ${ }^{1,2}$,
Wanying $\mathrm{Li}^{2}$, Zhaohui Hu ${ }^{5}$, Xiaoping $\mathrm{Li}^{* *}$, Qiang Liu ${ }^{1 *}$, Rilige Wu ${ }^{7 *}$ and Chengliang Yin
${ }^{1}$ Department of Orthopedics, Xianyang Central Hospital, Xianyang, China, ${ }^{2}$ Clinical Medical Research Center, Xianyang Central Hospital, Xianyang, China, ${ }^{3}$ Department of Spine Surgery, Second Affiliated Hospital of Dalian Medical University, Dalian, China, ${ }^{4}$ Department of Orthopaedics, The Second Hospital of Jilin University, Changchun, China, ${ }^{5}$ Department of Spine Surgery, Liuzhou People's Hospital, Liuzhou, China, ${ }^{6}$ Shulan International Medical College, Zhejiang Shuren University, Hangzhou, China, ${ }^{7}$ College of Information and Electrical Engineering, China Agricultural University, Beijing, China, ${ }^{8}$ Faculty of Medicine, Macau University of Science and Technology, Macau, Macao SAR, China

Background: This study aimed to construct a clinical prediction model for osteosarcoma patients to evaluate the influence factors for the occurrence of lymph node metastasis (LNM).

Methods: In our retrospective study, a total of 1,256 patients diagnosed with chondrosarcoma were enrolled from the SEER (Surveillance, Epidemiology, and End Results) database (training cohort, $n=1,144$ ) and multicenter dataset (validation cohort, $n=112$ ). Both the univariate and multivariable logistic regression analysis were performed to identify the potential risk factors of LNM in osteosarcoma patients. According to the results of multivariable logistic regression analysis, A nomogram were established and the predictive ability was assessed by calibration plots, receiver operating characteristics (ROCs) curve, and decision curve analysis (DCA). Moreover, Kaplan-Meier plot of overall survival (OS) was plot and a web calculator visualized the nomogram.

Results: Five independent risk factors [chemotherapy, surgery, lung metastases, lymphatic metastases (M-stage) and tumor size (T-stage)] were identified by multivariable logistic regression analysis. What's more, calibration plots displayed great power both in training and validation group. DCA presented great clinical utility. ROCs curve provided the predictive ability in the training cohort $(A \cup C=0.805)$ and the validation cohort (AUC $=0.808$ ). Moreover, patients in LNN group had significantly better survival than that in LNP group both in training and validation group.

Conclusion: In this study, we constructed and developed a nomogram with risk factors, which performed well in predicting risk factors of LNM in osteosarcoma patients. It may give a guide for surgeons and oncologists to optimize individual treatment and make a better clinical decision.

Keywords: nomogram, SEER, osteosarcoma, lymph node metastases, multicenter 


\section{INTRODUCTION}

Osteosarcoma is a common malignant bone tumor. The primary treatment consisting of neoadjuvant therapy, surgery and postoperative chemotherapy have resulted in the 5-year overall survival rate of $\sim 60 \%(1,2)$. However, even with the treatment of surgery and chemotherapy, the prognosis for patients with metastatic osteosarcoma remains dismal (3, 4).The lung metastases, the primary target of metastasis in osteosarcoma, has five-year survival rates of $\sim 30 \%(5,6)$. In extrapulmonary metastatic osteosarcoma, patients with lymph node metastases (LNM) have worse clinical outcomes, with fiveyear survival rates of $10 \%$ (7). However, only $3 \%$ of patients with osteosarcoma are diagnosed with LNM, leading to the lack of adequate clinical data for exploring osteosarcoma LNM (8).Therefore, a population-based study to assess the LNM in osteosarcoma is imminent.

Furthermore, disease forecasting is a vital part of the medical research (9-18). As a visual prediction tool, nomogram lists each variable separately and assigns a corresponding score for each status (19). Based on these considerations, we mined the Surveillance, Epidemiology, and End Results (SEER) database to construct the nomogram and used data from four academic hospitals for independent validation. This study contributes to providing more personalized guidance for patient care and improving patients' prognosis.

\section{MATERIALS AND METHODS}

\section{Data Collection}

In the present study, patients diagnosed with osteosarcoma between 2010 and 2016 were collected. The training group were extracted from the Surveillance, Epidemiology, and End Results (SEER) database with the SEER * Stat software version 8.3.6. And the third edition of the International Taxonomy of Oncology (ICDO-3), morphological code (9220) was used to identify osteosarcoma. The exclusion criteria of the training group were as follows: (1) patients with no positive pathology; (2) patients with unknown lymph node status and survival time; (3) more than one primary tumor.

Data of the validation group were obtained from four academic institutions, the Second Affiliated Hospital of Jilin University, the Second Affiliated Hospital of Dalian Medical University, the Liuzhou People's Hospital affiliated to Guangxi Medical University, and the Xianyang Central Hospital. And during the period of investigation, each center was responsible for the acquisition of data by three investigators. Two investigators were responsible for data extraction and the accuracy check was conducted by the third investigator. The exclusion criteria were consistent with the training group. For multicenter data, the study was approved by the ethics review committee of four medical institutions in China, the Second Affiliated Hospital of

Abbreviations: SEER, Surveillance, Epidemiology, and End Results database; ROC, receiver operating characteristic; DCA, decision curve analysis; CI, confidence interval; AUC, area under the curve; LNN, lymph node negative; LNM, lymph node metastases; ICD, International Classification of Diseases; SD, standard deviation; KM curves, Kaplan-Meier curves; OR, odds ratio.
Jilin University, the Second Affiliated Hospital of Dalian Medical University, Liuzhou People's Hospital, and Xianyang Central Hospital (No. 2021-00-22) and was conducted in accordance with the guidelines of the Helsinki Declaration.

Demographic and clinical variables, including race, age, survival time, sex, primary site, grade, laterality, tumor size (T-stage), distance metastases (M-stage), surgery, radiation, chemotherapy, bone metastases and lung metastases were considered in this study.

\section{Statistical Analysis}

Continuous variables were expressed as mean \pm standard deviation (SD), and categorical variables were expressed as frequency (proportions). The Student's $t$-test, Chi-square tests and Mann-Whitney tests were applied to continuous variables and categorical variables respectively with IBM SPSS Statistics version 26.0 (SPSS Inc., Chicago, Illinois, USA). Risk factors of the osteosarcoma were assessed using logistic regression. All analyzes were performed using R software version 3.6.2 (http:// www.r-project.org) including multiple $\mathrm{R}$ packages (Including regplot, rms, rmda and pROC). $P$ values $<0.05$ were considered statistically significant, and confidence intervals (CIs) were expressed as $95 \%$ confidence levels.

\section{Construction, Validation and Clinical Utility of a Nomogram}

The following variables were included in the univariate logistic regression analysis: race, age, survival time, sex, primary site, grade, laterality, tumor size, lymphatic metastasis, surgery, radiation, chemotherapy, bone metastases and lung metastases. According to the result of the univariate logistic regression analysis with the $P$ value $<0.05$, we performed the multivariable logistic regression analysis. And the Nomogram was constructed based on the results of multivariable logistic regression analysis with the $P$ value $<0.05$. Calibration plot and receiver operating characteristic (ROC) curves were used to evaluate the prediction performance of the nomogram. The higher the area under the curves (AUC) of ROC indicated the better model performance. In addition, the decision curve analysis (DCA) was used to evaluate the clinical utility of nomogram in decision-making. Based on the established nomogram, an interactive convenient web calculator was provided (https://drliwenle.shinyapps.io/LMOOapp/).

\section{RESULTS}

\section{Demographic Baseline Characteristics}

As shown in Table 1, a total of 1,256 patients were enrolled. There was no statistically significant difference between the training group $(n=1,144)$ and validation group $(n=112)$ except the Race $(P<0.001)$ and Chemotherapy $(P=0.017)$.

Additionally, these patients were divided into two subgroups according to the LNM in Table 2. There were no significant differences in race, sex, laterality and radiation between the lymph node negative group (LNN, $n=1,104$ ) and the lymph node positive (or unable to evaluate) group (LNP, $n=152$ ). However, the other characteristics showed significant differences between the two groups. 
TABLE 1 | Baseline data table of the training group and the validation group.

\begin{tabular}{|c|c|c|c|c|c|}
\hline Variable & level & $\begin{array}{c}\text { Overall } \\
(N=1,256)\end{array}$ & $\begin{array}{c}\text { SEER data } \\
\text { (Training group, } \\
N=1,144 \text { ) }\end{array}$ & $\begin{array}{l}\text { Multicenter data } \\
\quad \text { (validation } \\
\text { group, } N=112 \text { ) }\end{array}$ & $p$ \\
\hline \multirow[t]{3}{*}{ Race (\%) } & Black & $168(13.4)$ & $168(14.7)$ & $0(0.0)$ & $<0.001$ \\
\hline & Other & $228(18.2)$ & $116(10.1)$ & $112(100.0)$ & \\
\hline & White & $860(68.5)$ & $860(75.2)$ & $0(0.0)$ & \\
\hline Age [mean (SD)] & NA & $33.31(24.31)$ & $33.47(24.26)$ & $31.62(24.88)$ & 0.443 \\
\hline Times [mean (SD)] & NA & $29.93(22.69)$ & $29.91(22.54)$ & $30.10(24.24)$ & 0.933 \\
\hline \multirow[t]{2}{*}{$\operatorname{Sex}(\%)$} & Female & $573(45.6)$ & $521(45.5)$ & $52(46.4)$ & 0.936 \\
\hline & Male & $683(54.4)$ & $623(54.5)$ & $60(53.6)$ & \\
\hline \multirow[t]{3}{*}{ Primary.Site (\%) } & Axis bone & $336(26.8)$ & $309(27.0)$ & $27(24.1)$ & 0.349 \\
\hline & Limb bone & $817(65.0)$ & $738(64.5)$ & $79(70.5)$ & \\
\hline & Other & $103(8.2)$ & $97(8.5)$ & $6(5.4)$ & \\
\hline \multirow[t]{5}{*}{ Grade (\%) } & Moderately differentiated & 41 (3.3) & 41 (3.6) & $0(0.0)$ & 0.124 \\
\hline & Poorly differentiated & $302(24.0)$ & $279(24.4)$ & $23(20.5)$ & \\
\hline & Undifferentiated; anaplastic & $560(44.6)$ & $511(44.7)$ & $49(43.8)$ & \\
\hline & Unknown & $324(25.8)$ & $287(25.1)$ & 37 (33.0) & \\
\hline & Well-differentiated & $29(2.3)$ & $26(2.3)$ & $3(2.7)$ & \\
\hline \multirow[t]{3}{*}{ Laterality (\%) } & Left & $537(42.8)$ & $494(43.2)$ & $43(38.4)$ & 0.08 \\
\hline & Not a paired site & 173 (13.8) & $163(14.2)$ & $10(8.9)$ & \\
\hline & Right & 546 (43.5) & $487(42.6)$ & $59(52.7)$ & \\
\hline \multirow[t]{4}{*}{$\mathrm{T}(\%)$} & $\mathrm{T} 1$ & 426 (33.9) & 388 (33.9) & 38 (33.9) & 0.294 \\
\hline & $\mathrm{T} 2$ & 569 (45.3) & $523(45.7)$ & $46(41.1)$ & \\
\hline & T3 & $42(3.3)$ & $35(3.1)$ & 7 (6.2) & \\
\hline & TX & $219(17.4)$ & 198 (17.3) & $21(18.8)$ & \\
\hline \multirow[t]{2}{*}{ M (\%) } & MO & $976(77.7)$ & $892(78.0)$ & $84(75.0)$ & 0.547 \\
\hline & M1 & 280 (22.3) & $252(22.0)$ & $28(25.0)$ & \\
\hline \multirow[t]{2}{*}{ Surgery (\%) } & No & $254(20.2)$ & $230(20.1)$ & $24(21.4)$ & 0.834 \\
\hline & Yes & 1002 (79.8) & 914 (79.9) & 88 (78.6) & \\
\hline \multirow[t]{2}{*}{ Radiation (\%) } & No & 1103 (87.8) & 999 (87.3) & 104 (92.9) & 0.119 \\
\hline & Yes & $153(12.2)$ & $145(12.7)$ & $8(7.1)$ & \\
\hline \multirow[t]{2}{*}{ Chemotherapy (\%) } & No & $274(21.8)$ & $260(22.7)$ & $14(12.5)$ & 0.017 \\
\hline & Yes & $982(78.2)$ & $884(77.3)$ & $98(87.5)$ & \\
\hline \multirow[t]{3}{*}{ Bone metastases (\%) } & No & 1146 (91.2) & 1044 (91.3) & $102(91.1)$ & 0.981 \\
\hline & Unknown & $52(4.1)$ & $47(4.1)$ & $5(4.5)$ & \\
\hline & Yes & 58 (4.6) & 53 (4.6) & $5(4.5)$ & \\
\hline \multirow[t]{3}{*}{ Lung metastases (\%) } & No & 988 (78.7) & 901 (78.8) & 87 (77.7) & 0.931 \\
\hline & Unknown & 48 (3.8) & 44 (3.8) & $4(3.6)$ & \\
\hline & Yes & 220 (17.5) & $199(17.4)$ & $21(18.8)$ & \\
\hline
\end{tabular}

\section{Univariate and Multivariable Logistic Regression Results}

According to the univariate logistics regression analysis, we identified 10 prognostic factors including age, survival time, primary site, laterality, tumor size, lymph metastasis, surgery, chemotherapy, bone metastases and lung metastases in the training set $(P<0.05)$ (Table 3). Then, based on the above result, applying the multivariable logistics regression analysis, we figured out five independent prognostic factors including T-stage [TX: odds ratio (OR) 3.602,95\%CI 1.710-5.483, $P<$ 0.001 ], M-stage (M1: OR $=2.890,1.463-5.709, P<0.01$ ), surgery (Yes: $\mathrm{OR}=0.418,0.247-0.706, P<0.01$ ), Chemotherapy
(Yes: $\mathrm{OR}=0.475,0.267-0.819, P<0.01$ )and Lung metastases (Unknown: $\mathrm{OR}=9.407,1.955-45.261, P<0.01$ ) (Table 3).

\section{Construction and Validation of Nomogram for Chondrosarcoma Patients}

The nomogram contained five risk factors confirmed to be statistically significant by logistic regression analysis, including T-stage, M-stage, surgery, chemotherapy and lung metastases (Figure 1A). Calibration chart of nomogram showed a good consistency in the training and validation groups (Figures 1B,C). The AUC values of nomogram were 0.805 (95\% CI $0.781-$ $0.827)$ and $0.808(95 \%$ CI $0.723-0.877)$ in the training group and 
TABLE 2 | Patient baseline table of lymphatic metastases.

\begin{tabular}{|c|c|c|c|c|c|}
\hline & Level & $\begin{array}{c}\text { Overall } \\
(N=1,256)\end{array}$ & No $(N=1,104)$ & $\begin{array}{c}\text { Yes/Unable to } \\
\text { evaluate } \\
(N=152)\end{array}$ & $p$ \\
\hline \multirow[t]{2}{*}{ Category (\%) } & Multicenter data (validation group) & $112(8.9)$ & $93(8.4)$ & $19(12.5)$ & 0.133 \\
\hline & SEER data (Training group) & $1144(91.1)$ & $1011(91.6)$ & $133(87.5)$ & \\
\hline Times [mean (SD)] & NA & $29.93(22.69)$ & $30.95(22.70)$ & $22.45(21.18)$ & $<0.001$ \\
\hline \multirow[t]{3}{*}{ Race (\%) } & black & $168(13.4)$ & $145(13.1)$ & $23(15.1)$ & 0.417 \\
\hline & Other & $228(18.2)$ & $196(17.8)$ & $32(21.1)$ & \\
\hline & White & $860(68.5)$ & $763(69.1)$ & $97(63.8)$ & \\
\hline Age [mean (SD)] & NA & $33.31(24.31)$ & $32.48(23.79)$ & $39.35(27.16)$ & 0.001 \\
\hline \multirow[t]{2}{*}{$\operatorname{Sex}(\%)$} & Female & $573(45.6)$ & $498(45.1)$ & 75 (49.3) & 0.37 \\
\hline & Male & $683(54.4)$ & 606 (54.9) & $77(50.7)$ & \\
\hline \multirow[t]{3}{*}{ Primary.Site (\%) } & Axis bone & $336(26.8)$ & $282(25.5)$ & $54(35.5)$ & 0.027 \\
\hline & Limb bone & $817(65.0)$ & $732(66.3)$ & $85(55.9)$ & \\
\hline & Other & $103(8.2)$ & $90(8.2)$ & $13(8.6)$ & \\
\hline \multirow[t]{5}{*}{ Grade (\%) } & Moderately differentiated & $41(3.3)$ & $39(3.5)$ & $2(1.3)$ & $<0.001$ \\
\hline & Poorly differentiated & $302(24.0)$ & $273(24.7)$ & $29(19.1)$ & \\
\hline & Undifferentiated; anaplastic & $560(44.6)$ & $508(46.0)$ & $52(34.2)$ & \\
\hline & Unknown & $324(25.8)$ & $257(23.3)$ & $67(44.1)$ & \\
\hline & Well-differentiated & $29(2.3)$ & $27(2.4)$ & $2(1.3)$ & \\
\hline \multirow[t]{3}{*}{ Laterality (\%) } & Left & $537(42.8)$ & $483(43.8)$ & $54(35.5)$ & 0.104 \\
\hline & Not a paired site & $173(13.8)$ & $146(13.2)$ & $27(17.8)$ & \\
\hline & Right & $546(43.5)$ & $475(43.0)$ & $71(46.7)$ & \\
\hline \multirow[t]{4}{*}{$\mathrm{T}(\%)$} & $\mathrm{T} 1$ & $426(33.9)$ & $400(36.2)$ & $26(17.1)$ & $<0.001$ \\
\hline & $\mathrm{T} 2$ & $569(45.3)$ & $526(47.6)$ & 43 (28.3) & \\
\hline & T3 & $42(3.3)$ & $36(3.3)$ & $6(3.9)$ & \\
\hline & TX & $219(17.4)$ & $142(12.9)$ & $77(50.7)$ & \\
\hline \multirow[t]{2}{*}{ M (\%) } & MO & $976(77.7)$ & $883(80.0)$ & $93(61.2)$ & $<0.001$ \\
\hline & M1 & $280(22.3)$ & $221(20.0)$ & $59(38.8)$ & \\
\hline \multirow[t]{2}{*}{ Surgery (\%) } & No & $254(20.2)$ & $179(16.2)$ & 75 (49.3) & $<0.001$ \\
\hline & Yes & 1002 (79.8) & 925 (83.8) & $77(50.7)$ & \\
\hline \multirow[t]{2}{*}{ Radiation (\%) } & No & $1103(87.8)$ & 975 (88.3) & $128(84.2)$ & 0.187 \\
\hline & Yes & $153(12.2)$ & $129(11.7)$ & $24(15.8)$ & \\
\hline \multirow[t]{2}{*}{ Chemotherapy (\%) } & No & $274(21.8)$ & $208(18.8)$ & $66(43.4)$ & $<0.001$ \\
\hline & Yes & $982(78.2)$ & $896(81.2)$ & $86(56.6)$ & \\
\hline \multirow[t]{3}{*}{ Bone metastases (\%) } & No & $1146(91.2)$ & $1046(94.7)$ & $100(65.8)$ & $<0.001$ \\
\hline & Unknown & $52(4.1)$ & $14(1.3)$ & $38(25.0)$ & \\
\hline & Yes & $58(4.6)$ & $44(4.0)$ & $14(9.2)$ & \\
\hline
\end{tabular}

the validation group respectively (Figures 2A,B). Furthermore, the ROC curve demonstrated a superior performance of the nomogram compared to the single variable, including chemotherapy ( $\mathrm{AUC}=0.631,95 \% \mathrm{CI} 0.602$ to 0.659 ), lung metastases $(0.697,0.669$ to 0.723$)$, M-stage ( $\mathrm{AUC}=0.592,95 \% \mathrm{CI}$ 0.563 to 0.621 ), surgery ( $\mathrm{AUC}=0.667,95 \% \mathrm{CI} 0.639$ to 0.694 ) and T-stage $(0.706,95 \% \mathrm{CI} 0.678$ to 0.732$)$. The statistical results of validation group were consistent with the training group as shown in Table 4. In addition, an online web calculator was designed (https://drliwenle.shinyapps.io/LMOOapp/).

\section{Clinical Applicability of the Nomogram}

The Kaplan-Meier survival curves of training group were plotted (Figure 3A). The results revealed that the overall survival (OS) significantly decreased in patients with LNP comparing with the LNN $(P<0.001)$. Moreover, the threshold about 0.1 to 0.9 had the maximum benefit range of the model as shown in the DCA curve (Figures 3C,D). The Kaplan-Meier survival curves of validation group displayed the same trend between the two groups $(\mathrm{P}<0.001)$ (Figure 3B).

\section{DISCUSSION}

Osteosarcoma metastases, which are typically secondary to hematogenous dissemination and the occurrence of lymph system is extremely rare, have been identified to be significantly associated with poor prognosis $(1-4,7,20,21)$. Comparing to 
TABLE 3 | Univariate and multifactorial logistic regression analysis of risk factors for Lymph node metastases in patients with osteosarcoma.

\begin{tabular}{|c|c|c|c|c|}
\hline Variables & Univariate OR $(95 \% \mathrm{Cl})$ & $p$ value & Multivariate OR $(95 \% \mathrm{Cl})$ & $p$ value \\
\hline Age (years) & $1.011(1.004-1.018)$ & $<0.01$ & $0.993(0.982-1.004)$ & 0.397 \\
\hline Survival time (month) & 0.983 (0.974-0.992) & $<0.001$ & $0.998(0.987-1.010)$ & 0.788 \\
\hline \multicolumn{5}{|l|}{ Race } \\
\hline White & Reference & Ref & Ref & Ref \\
\hline Black & $1.248(0.766-3.033)$ & 0.374 & / & / \\
\hline Other & $0.993(0.537-1.835)$ & 0.982 & / & / \\
\hline \multicolumn{5}{|l|}{ Sex } \\
\hline Male & Ref & Ref & Ref & Ref \\
\hline Female & $1.163(0.810-1.671)$ & 0.412 & 1 & I \\
\hline \multicolumn{5}{|l|}{ Primary site } \\
\hline Limb bones & Ref & Ref & Ref & Ref \\
\hline Axis of a bone & $1.675(1.133-2.478)$ & $<0.05$ & $0.946(0.517-1.731)$ & 0.857 \\
\hline Other & $1.286(0.671-2.466)$ & 0.449 & $1.840(0.806-4.198)$ & 0.148 \\
\hline \multicolumn{5}{|l|}{ Grade } \\
\hline Well-differentiated & Ref & Ref & Ref & Ref \\
\hline Moderately differentiated & $1.282(0.110-14.893)$ & 0.843 & / & / \\
\hline Poorly differentiated & 2.569 (0.334-19.741) & 0.364 & / & / \\
\hline Undifferentiated; anaplastic & $2.473(0.328-18.673)$ & 0.380 & / & / \\
\hline Unknown & $6.332(0.840-47.705)$ & 0.073 & / & / \\
\hline \multicolumn{5}{|l|}{ Laterality } \\
\hline Left & Ref & Ref & Ref & Ref \\
\hline Right & 1.395 (0.930-2.091) & 0.108 & $1.477(0.920-2.371)$ & 0.106 \\
\hline Other & $1.848(1.102-3.101)$ & $<0.05$ & $1.113(0.536-2.310)$ & 0.774 \\
\hline \multicolumn{5}{|l|}{$\mathbf{T}$} \\
\hline T1 & Ref & Ref & Ref & Ref \\
\hline T2 & $1.121(0.657-1.912)$ & 0.675 & $0.960(0.540-1.707)$ & 0.889 \\
\hline T3 & $2.528(0.900-7.101)$ & 0.078 & $1.473(0.476-4.559)$ & 0.502 \\
\hline TX & $7.933(4.780-13.167)$ & $<0.001$ & $3.062(1.710-5.483)$ & $<0.001$ \\
\hline \multicolumn{5}{|l|}{ M } \\
\hline MO & Ref & Ref & Ref & Ref \\
\hline M1 & 2.506 (1.710-3.673) & $<0.001$ & $2.890(1.463-5.709)$ & $<0.01$ \\
\hline \multicolumn{5}{|l|}{ Surgery } \\
\hline No & Ref & Ref & Ref & Ref \\
\hline Yes & $0.197(0.135-0.287)$ & $<0.001$ & $0.418(0.247-0.706)$ & $<0.01$ \\
\hline \multicolumn{5}{|l|}{ Radiation } \\
\hline No & Ref & Ref & Ref & Ref \\
\hline Yes & $1.524(0.936-2.481)$ & 0.091 & / & / \\
\hline \multicolumn{5}{|l|}{ Chemotherapy } \\
\hline No & Ref & Ref & Ref & Ref \\
\hline Yes & $0.289(0.199-0.421)$ & $<0.001$ & $0.475(0.276-0.819)$ & $<0.01$ \\
\hline \multicolumn{5}{|l|}{ Bone metastases } \\
\hline No & Ref & Ref & Ref & Ref \\
\hline Yes & $3.260(1.652-6.436)$ & $<0.01$ & $1.257(0.570-2.772)$ & 0.571 \\
\hline Unknown & 32.490 (16.267-64.892) & $<0.001$ & 2.159 (0.483-9.659) & 0.314 \\
\hline \multicolumn{5}{|l|}{ Lung metastases } \\
\hline No & Ref & Ref & Ref & Ref \\
\hline Yes & $2.938(1.887-4.573)$ & $<0.001$ & $0.902(0.449-1.812)$ & 0.771 \\
\hline Unknown & 45.225 (21.360-95.754) & $<0.001$ & $9.407(1.955-45.261)$ & $<0.01$ \\
\hline
\end{tabular}

the most common lung metastases, osteosarcoma patients with lymph node involvement have a worse prognosis, suggesting that the invasion of the lymph nodes is an important indicator for the assessment of malignancy stage and the selection of a correct treatment protocol $(22,23)$.Meanwhile, studies points out that FDG-PET and ${ }^{99} \mathrm{~m}$ Tc-labeled biomineralization 

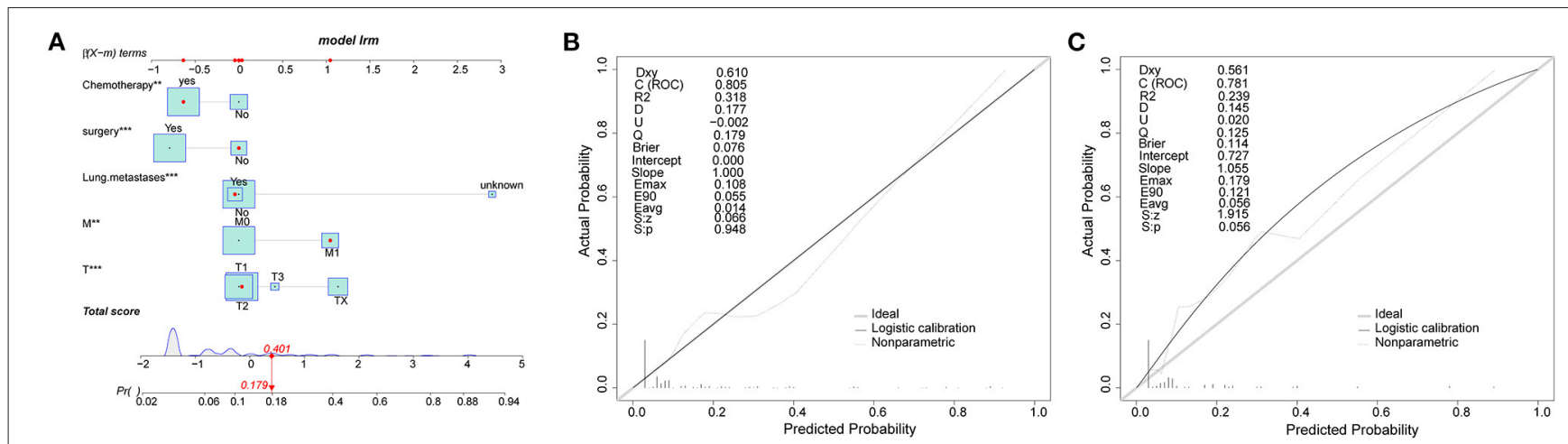

FIGURE 1 | (A) Nomogram for osteosarcoma patients. (B,C) are training cohorts and the validation cohorts calibration diagram respectively, which indicate good consistency.

A

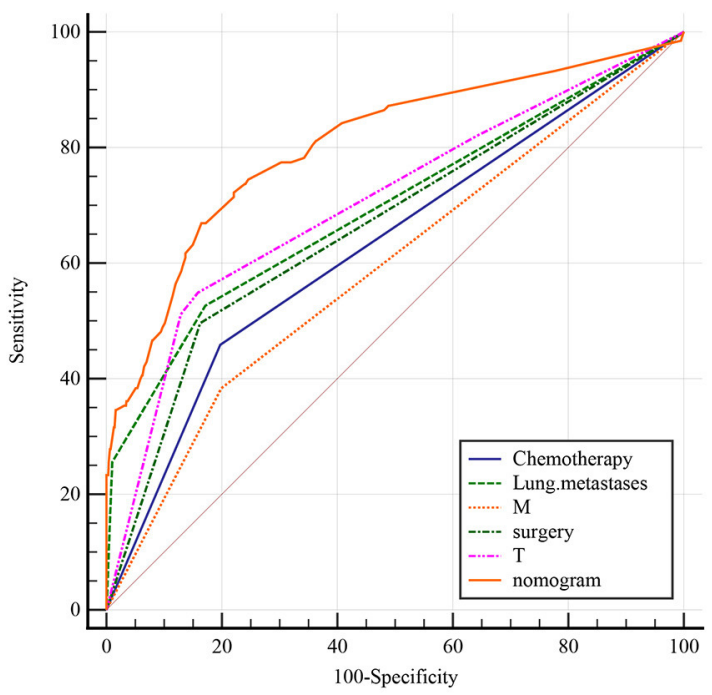

B

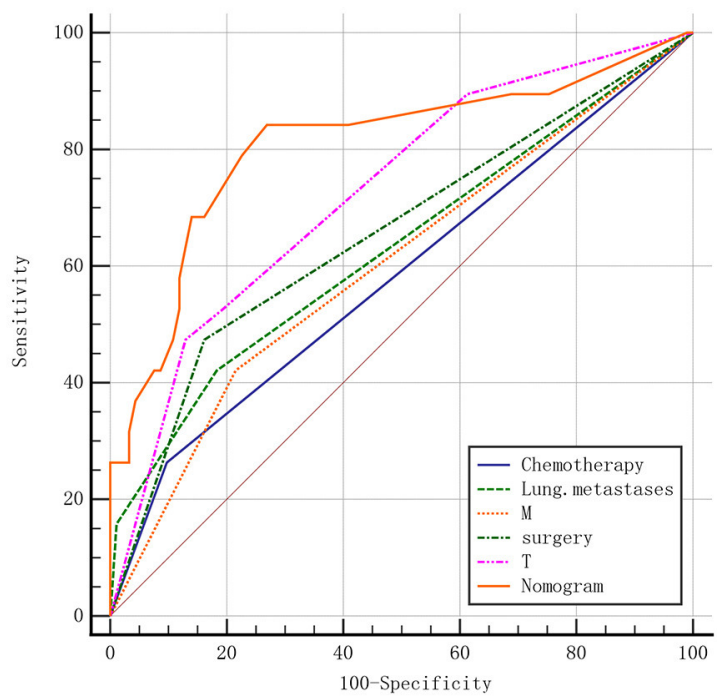

FIGURE 2 | ROC curves for the training and validation group. (A) training group; (B) validation group.

TABLE 4 | AUC of training group and validation group.

\begin{tabular}{|c|c|c|c|c|c|c|}
\hline Variable & \multicolumn{3}{|c|}{ SEER data (Training group) } & \multicolumn{3}{|c|}{ Multicenter data (validation group) } \\
\hline Lung metastases & 0.697 & 0.0239 & 0.669 to 0.723 & 0.631 & 0.0644 & 0.535 to 0.721 \\
\hline M & 0.592 & 0.0221 & 0.563 to 0.621 & 0.603 & 0.062 & 0.506 to 0.694 \\
\hline Surgery & 0.667 & 0.0225 & 0.639 to 0.694 & 0.656 & 0.0619 & 0.561 to 0.743 \\
\hline
\end{tabular}

nanoprobe are effective in early diagnosis of metastatic lymph nodes in osteosarcoma (24-27). Therefore, real-time lymph node surveillance and radical treatment for osteosarcoma patients with a high risk of lymph node metastasis will improve patient survival (8). In this study, we identified five independent risk factors associated with LNM and provided a convenient nomogram prediction model and a web calculator on the basis of the model. 


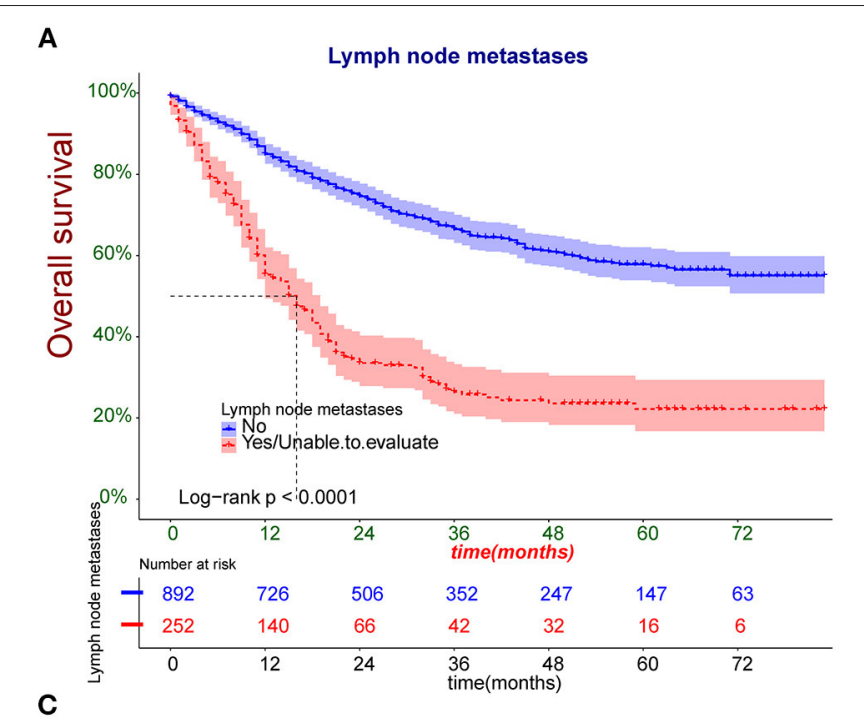

C

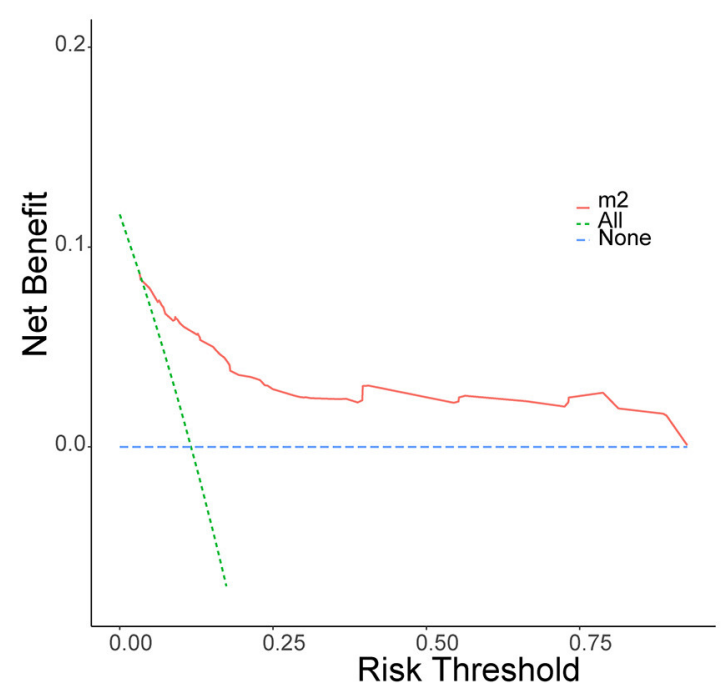

$\mathbf{B}$
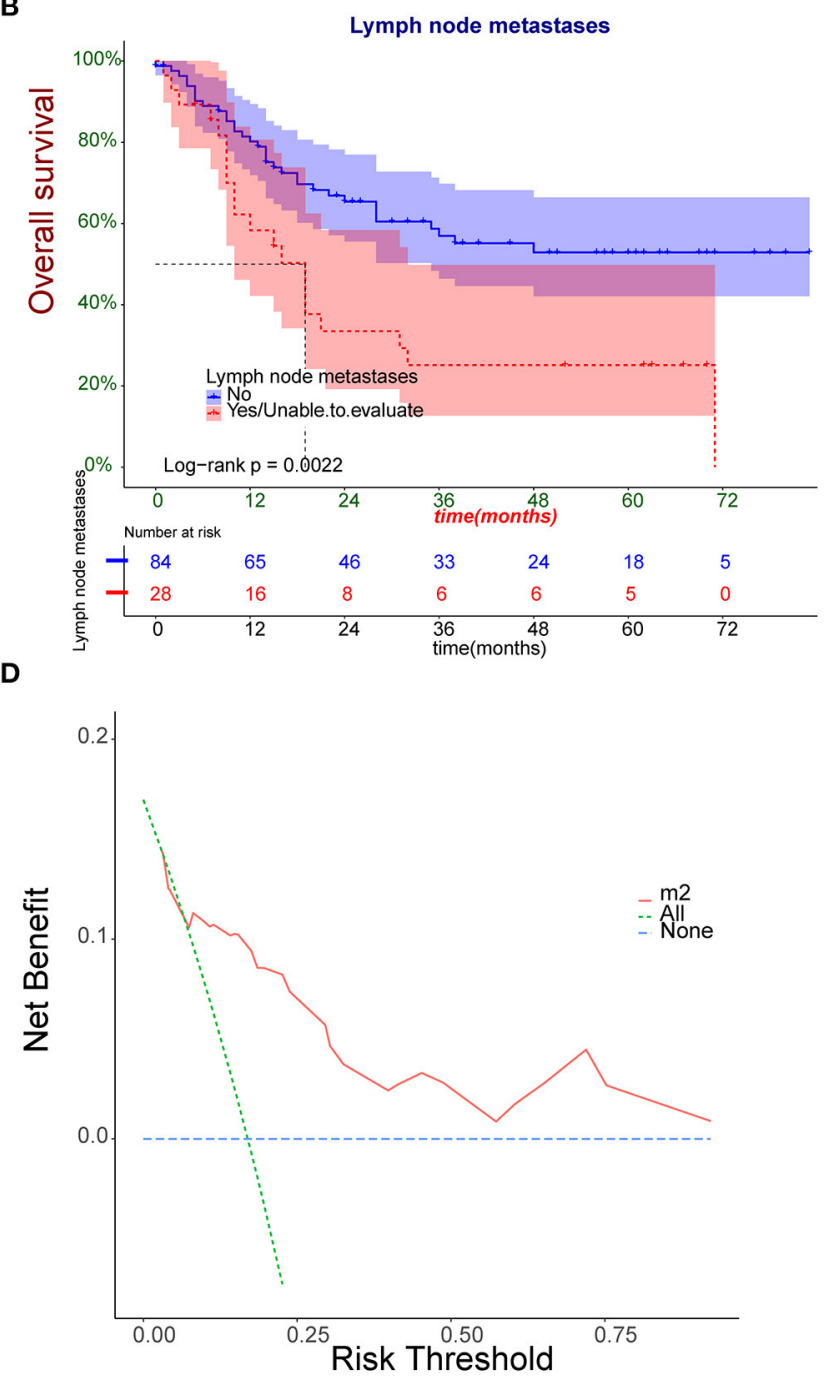

FIGURE 3 | (A,B) The Kaplan-Meier survival analysis patients with osteosarcoma in training and validation group. (C,D) Nomogram decision curve (DCA) of the training and validation group.

Surgery and chemotherapy are the most reliable and effective treatment options for prolonging the lives of patients and have been followed in several clinical trials (28-30). According to the multivariable logistic analysis, chemotherapy and surgery were crucial prognostic indicators. Surgery and chemotherapy contributed to improve patient prognosis, which were consistent with the result of the KM survival curve. This suggests that timely and effective treatment plays an important role in controlling lymph node metastasis and enhancing OS $(15,17,31,32)$. Unfortunately, the relationship between radiotherapy and lymph node metastasis has not been proved. A possible reason for these results would be the uncertainty of radiation therapy in cancer treatment along with the development of radiation-associated osteosarcoma make the safety of chemotherapy need to be further ensured (33, $34)$.
Due to the lack of lymphatic drainage in normal cortical bone and spongy bone, LNM is rare in bone sarcomas (35, 36).Regional lymph node involvement in osteosarcoma may be owing to the infiltration of the enlarged tumor parenchyma into the periphery, such as the joint capsule or synovium, leading to dissemination into the lymphatic system $(37,38)$.Our study reported T-stage as a significant predictor of LNM, which was also consistent with previous findings (7).Meanwhile, because of the fact that the peak incidence of the osteosarcoma is 1519 years of age, more than $80 \%$ of patients achieve limb salvage through surgery $(4,28,38)$.In this respect, the surgeons need pay more attention to the status of the regional lymph nodes during the resection of larger osteosarcoma in order to eradicate the sarcoma and preserve the limb. M-stage indicates distant metastasis in osteosarcoma, and it is also a risk factor associated with LNM. In the study by Thampi et al. osteosarcoma lymphatic 
metastasis was significantly associated with distant metastasis (7).Furthermore, since the lung was the most important target organ for distant metastasis in osteosarcoma, we separated this variable from distant metastasis to emphasize the significant role played by lung metastasis in assessing lymph node status. Patients with lung metastases express the characteristic biomarker, such as KEAP, Matrix-Gla and Rab22a (39-41). Considering the intense correlation we found between lung metastasis and lymph node involvement, these reported biomarkers would be triggers for LNM. Therefore, this provides inspiration to further molecular biology studies focusing on lymph node metastasis in osteosarcoma.

We constructed a novel nomogram to assess the risk of developing lymph node metastasis in osteosarcoma, and the discriminatory of any individual predictor was inferior to that of nomogram, suggesting that the nomogram model indicated promising prospects for tumor surveillance and clinical decision making. Although some predictive nomograms have been reported in previous studies, our study complements previous work. Compared to Dong et al.'s study, external independent validation consisting of multiple academic centers is a prominent feature of this study, and the inclusion of multiple ethnic groups enhances the credibility of the results (8). Moreover, we provide a convenient and digital prediction tool for users. By analyzing the clinical characteristics and associated risk factors, we improve the prediction of lymph node metastasis risk in osteosarcoma and provide a basis for individualized treatment and follow-up strategies. The web-based calculator constructed in this study is an easy-to-use clinical tool that helps to promote personalized treatment.

Finally, one obvious limitation in this study was that the statistically significant difference between the training group and validation groups in chemotherapy may have an influence on the results. Another limitation was the lack of a more thoroughly analysis due to the inadequacy of systematic and prospective data.

\section{CONCLUSION}

In conclusion, we constructed a novel nomogram model to predict risk factors for osteosarcoma patients developing LNM, including T-stage, M-stage, surgery, chemotherapy and lung metastases based on epidemiological characteristics obtained from the SEER database and the multicenter hospitals. By

\section{REFERENCES}

1. Marina N, Smeland S, Bielack S, Bernstein M, Jovic G, Hook J, et al. "MAPIE vs MAP as postoperative chemotherapy in patients with a poor response to preoperative chemotherapy for newly-diagnosed osteosarcoma: results from EURAMOS-1 (Paper 032)," in Presented at Connective Tissue Oncology Society (2014).

2. Tian Z, Niu X, Yao W. Receptor tyrosine kinases in osteosarcoma treatment: which is the key target? Front Oncol. (2020) 10:1642. doi: 10.3389/fonc.2020.01642

3. Kager L, Zoubek A, Pötschger U, Kastner U, Flege S, Kempf-Bielack B, et al. Primary metastatic osteosarcoma: presentation and outcome of patients combining DCA curve, ROC curve, KM curve, web calculator and external validation, our nomogram provided an accurate assessment for individualized risk of lymph node metastasis which was helpful for clinicians to make better surgery decisions.

\section{DATA AVAILABILITY STATEMENT}

The raw data supporting the conclusions of this article will be made available by the authors without undue reservation.

\section{ETHICS STATEMENT}

The SEER database is a comprehensive data source developed based on population data and updated annually since its launch in 1973. It is public and identifiably accessible that data analysis is treated as non-human subjects by the Office for Human Research Protections. As such, no institutional review board approval and informed consent were required. For multicenter data, the study was approved by the ethics review committee of four medical institutions in China, the Second Affiliated Hospital of Jilin University, the Second Affiliated Hospital of Dalian Medical University, 291 Liuzhou People's Hospital, and Xianyang Central Hospital (No. 2021-0022) and was conducted in accordance with the guidelines of the Helsinki.

\section{AUTHOR CONTRIBUTIONS}

CY, RW, QL, and XL designed the study. WeL and SD performed the study, analyzed the data, and wrote the manuscript. BW and HW provided the expert consultations and clinical suggestions. $\mathrm{CX}$ and $\mathrm{KZ}$ conceived of the study, participated in its design and coordination. WaL and $\mathrm{ZH}$ helped to draft the manuscript. All authors reviewed the final version of the manuscript.

\section{ACKNOWLEDGMENTS}

We thank China's multicenter for providing us individual patient data. The multicenter data were obtained from four medical institutions in China, the Second Affiliated Hospital of Jilin University, the Second Affiliated Hospital of Dalian Medical University, Liuzhou People's Hospital, and Xianyang Central Hospital. treated on neoadjuvant Cooperative Osteosarcoma Study Group protocols. $J$ Clin Oncol. (2003) 21:2011-8. doi: 10.1200/JCO.2003.08.132

4. Hattori H, Yamamoto K. Lymph node metastasis of osteosarcoma. J Clin Oncol. (2012) 30:345-9. doi: 10.1200/JCO.2012.42.3384

5. Kempf-Bielack B. Osteosarcoma relapse after combined modality therapy: an analysis of unselected patients in the Cooperative Osteosarcoma Study Group (COSS). J Clin Oncol. (2005) 23:559-68. doi: 10.1200/JCO.2005. 04.063

6. Zheng B, Zhou C, Qu G, Ren C, Yue B. VEGFR2 promotes metastasis and PD-L2 expression of human osteosarcoma cells by activating the STAT3 and RhoA-ROCK-LIMK2 pathways. Front Oncol. (2020) 10:543562. doi: $10.3389 /$ fonc. 2020.543562 
7. Thampi S, Matthay KK, Goldsby R, Dubois SG. Adverse impact of regional lymph node involvement in osteosarcoma. Eur J Cancer. (2013) 49:34716. doi: 10.1016/j.ejca.2013.06.023

8. Dong Y, Wu W, Kang H, Xiong W, Li F. Risk factors of regional lymph node (RLN) metastasis among patients with bone sarcoma and survival of patients with RLN-positive bone sarcoma. Ann Transl Med. (2021) 9:48. doi: 10.21037/atm-20-4681

9. Kumar R, Tripathi R, Marchang N, Srivastava G, Gadekallu TR, Xiong NN. A secured distributed detection system based on IPFS and blockchain for industrial image and video data security. J Parallel Distributed Comput. (2021) 152:128-43. doi: 10.1016/j.jpdc.2021.02.022

10. Agrawal S, Sarkar S, Srivastava G, Maddikunta P, Gadekallu TR. Genetically optimized prediction of remaining useful life. Sustain Comput. (2021) 31:100565. doi: 10.1016/j.suscom.2021.100565

11. Naeem A, Javed AR, Rizwan M, Abbas S, Gadekallu TR. DARE-SEP: a hybrid approach of distance aware residual energy-efficient SEP for WSN. IEEE Trans Green Commun Netw. (2021) 5:611-21. doi: 10.1109/TGCN.2021.3067885

12. Gadekallu TR, Alazab M, Kaluri R, Maddikunta P, Parimala M. Hand gesture classification using a novel CNN-crow search algorithm. Complex Intell Syst. (2021) 7:1855-68. doi: 10.1007/s40747-021-00324-x

13. Gadekallu TR, Manoj MK, Sivarama KS, Kumar N, Bhattacharya S. Blockchain based attack detection on machine learning algorithms for IoT based E-health applications. IEEE Internet Things Mag. (2020) 4:3033. doi: 10.1109/IOTM.1021.2000160

14. Tang Z, Zhu R, Lin P, He J, Wang H, Huang Q, et al. A hardware friendly unsupervised memristive neural network with weight sharing mechanism. Neurocomputing. (2019) 332:193-202. doi: 10.1016/j.neucom.2018.12.049

15. Wu EQ, Hu D, Deng PY, Tang Z, Ren H. Nonparametric bayesian prior inducing deep network for automatic detection of cognitive status. IEEE Trans Cybern. (2020) 51:5483-96. doi: 10.1109/TCYB.2020.2977267

16. Tang Z, Chen $\mathrm{Y}$, Ye S, Hu R, Chang S. Fully Memristive spiking-neuron learning framework and its applications on pattern recognition and edge detection. Neurocomputing. (2020) 403:80-7. doi: 10.1016/j.neucom.2020.04.012

17. Wu EQ, Deng PY, Qu XY, Tang Z, Sheng RSF. Detecting fatigue status of pilots based on deep learning network using EEG signals. IEEE Trans Cogn Dev Syst. (2020) 13:575-85. doi: 10.1109/TCDS.2019.2963476

18. Tang Z, Zhu R, Hu R, Chen Y, Chang S. A multilayer neural network merging image preprocessing and pattern recognition by integrating diffusion and drift memristors. IEEE Trans Cogn Dev Syst. (2020) 13:64556. doi: 10.1109/TCDS.2020.3003377

19. Balachandran VP, Gonen M, Smith JJ, Dematteo RP. Nomograms in oncology: more than meets the eye. Lancet Oncol. (2015) 16:e173e80. doi: 10.1016/S1470-2045(14)71116-7

20. Meazza C, Scanagatta P. Metastatic osteosarcoma: a challenging multidisciplinary treatment. Expert Rev Anticancer Ther. (2016) 16:543-56. doi: 10.1586/14737140.2016.1168697

21. Wang T, Quan Y, Shen XS, Gadekallu TR, Wang W, Dev K, et al. Privacyenhanced retrieval technology for the cloud-assisted internet of things. IEEE Trans Ind Inform. (2021) 14:1551-3203. doi: 10.1109/TII.2021.3103547

22. Alitalo K. The lymphatic vasculature in disease. Nat Med. (2011) 17:137180. doi: $10.1038 / \mathrm{nm} .2545$

23. Wang W, Qiu C, Yin Z, Srivastava G, Gadekallu TR, Alsolami F, et al. Blockchain and PUF-based lightweight authentication protocol for wireless medical sensor networks. IEEE Internet Things J. (2021) 11. doi: 10.1109/JIOT.2021.3117762

24. Xu Z, Wang Y, Han J, Xu Q, Ren J, Xu J, et al. Noninvasive multimodal imaging of osteosarcoma and lymph nodes using a 99mTc-labeled biomineralization nanoprobe. Anal Chem. (2018) 90:4529-34. doi: 10.1021/acs.analchem.7b04925

25. Volker T, Denecke T, Steffen I, Misch D, Schonberger S, Plotkin $\mathrm{M}$, et al. Positron emission tomography for staging of pediatric sarcoma patients: results of a prospective multicenter trial. J Clin Oncol. (2007) 25:5435. doi: 10.1200/JCO.2007.1 2.2473
26. Wang W, Fida MH, Lian Z, Yin Z, Pham QV, Gadekallu TR, et al. Secure-enhanced federated learning for AI-empowered electric vehicle energy prediction. IEEE Consum Electron Mag. (2021). doi: 10.1109/MCE.2021.31 16917

27. Xiong $H$, Jin $C$, Alazab $M$, Yeh KH, Wang $H$, Gadekallu TRR, et al. On the design of blockchain-based ECDSA with faulttolerant batch verication protocol for blockchain-enabled IoMT. IEEE J Biomed Health Inform. (2021). doi: 10.1109/JBHI.2021.31 12693

28. Ritter J, Bielack SS. Osteosarcoma. Ann Oncol. (2010) 21(Suppl 7):vii320-5. doi: 10.1093/annonc/ mdq276

29. Pramanik R, Agarwala S, Gupta YK, Thulkar S, Bakhshi S. Metronomic chemotherapy vs best supportive care in progressive pediatric solid malignant tumors: a randomized clinical trial. Jama Oncol. (2017) 3:1222-7. doi: 10.1001/jamaoncol.20 17.0324

30. Piperno-Neumann S, Le Deley MC, Rédini F, Pacquement H, Marec-Bérard $\mathrm{P}$, Petit $\mathrm{P}$, et al. Zoledronate in combination with chemotherapy and surgery to treat osteosarcoma (OS2006): a randomised, multicentre, open-label, phase 3 trial. Lancet Oncol. (2016) 17:1070-80. doi: 10.1016/S1470-2045(16)3 0096-1

31. Huang $\mathrm{Z}$, Hu C, Liu $\mathrm{K}$, Yuan L, Hu C. Risk factors, prognostic factors, and nomograms for bone metastasis in patients with newly diagnosed infiltrating duct carcinoma of the breast: a populationbased study. BMC Cancer. (2020) 20:1145. doi: 10.1186/s12885-020-0 7635-1

32. Wu EQ, Zhou MC, Hu D, Zhu L, Ren H. Self-paced dynamic infinite mixture model for fatigue evaluation of pilots' brains. IEEE Trans Cybern. (2020) 2168-267. doi: 10.1109/TCYB.2020.30 33005

33. Siontis BL, Mchugh JB, Roberts E, Zhao L, Chugh R Differential outcomes and biologic markers of radiationassociated vs. sporadic osteosarcoma: a single-institution experience. Front Oncol. (2020) 9:1523. doi: 10.3389/fonc.2019. 01523

34. Wu EQ, Zhu LM, Li GJ, Li HJ, Zhou GR. Nonparametric hierarchical hidden semi-markov model for brain fatigue behavior detection of pilots during flight. IEEE Trans Intell Transport Syst. (2021) 1-12. doi: 10.1109/TITS.2021.3052801

35. Cleary MX, Fayad LM, Ahlawat S. Popliteal lymph nodes in patients with osteosarcoma: are they metastatic? Skeletal Radiol. (2020) 49:1807-17. doi: 10.1007/s00256-020-0 3498-6

36. Wu EQ, Lin CT, Zhu LM, Tang ZR, Jie YW, Zhou GR. Fatigue detection of pilots' brain through brains cognitive map and multilayer latent incremental learning model. IEEE Trans Cybern. (2021) 1-13. doi: 10.1109/TCYB.2021.30 68300

37. Dirik Y, Çinar A, Yumrukçal F, Eralp L. Popliteal lymph node metastasis of tibial osteoblastic osteosarcoma. Int J Surg Case Rep. (2014) 5:840-4. doi: 10.1016/j.ijscr.2014. 09.029

38. Bielack SS, Kempf-Bielack B, Delling G, Exner GU, Flege S, Helmke $\mathrm{K}$, et al. Prognostic factors in high-grade osteosarcoma of the extremities or trunk: an analysis of 1,702 patients treated on neoadjuvant cooperative osteosarcoma study group protocols. J Clin Oncol. (2002) 20:776-90. doi: 10.1200/JCO.2002.2 0.3 .776

39. Xu H, Zhu X, Bao H, Shek TW, Huang Z, Wang Y, et al. Genetic and clonal dissection of osteosarcoma progression and lung metastasis. Int J Cancer. (2018) 143:1134-42. doi: 10.1002/ijc.31389

40. Zandueta C, Ormazábal C, Perurena N, Martínez-Canarias S, Zalacaín M, Julián MS, et al. Matrix-Gla protein promotes osteosarcoma lung metastasis and associates with poor prognosis. J Pathol. (2016) 239:43849. doi: $10.1002 /$ path. 4740 
41. Liao D, Zhong L, Yin J, Zeng C, Wang $\mathrm{X}$, Huang $\mathrm{X}$, et al. Chromosomal translocation-derived aberrant Rab22a drives metastasis of osteosarcoma. Nat Cell Biol. (2020) 22:868-81doi: 10.1038/s41556-020$0522-\mathrm{z}$

Conflict of Interest: The authors declare that the research was conducted in the absence of any commercial or financial relationships that could be construed as a potential conflict of interest.

Publisher's Note: All claims expressed in this article are solely those of the authors and do not necessarily represent those of their affiliated organizations, or those of the publisher, the editors and the reviewers. Any product that may be evaluated in this article, or claim that may be made by its manufacturer, is not guaranteed or endorsed by the publisher.

Copyright $\odot 2022$ Li, Dong, Wang, Wang, Xu, Zhang, Li, Hu, Li, Liu, Wu and Yin. This is an open-access article distributed under the terms of the Creative Commons Attribution License (CC BY). The use, distribution or reproduction in other forums is permitted, provided the original author(s) and the copyright owner(s) are credited and that the original publication in this journal is cited, in accordance with accepted academic practice. No use, distribution or reproduction is permitted which does not comply with these terms. 